\title{
Speaker'scorner
}

\section{Tackling inequalities in health: the need for building a systematic evidence base}

Socioeconomic inequalities in health have been demonstrated in all countries with available data, and evidence on determinants of these inequalities is accumulating. Our understanding of the problem has reached the point where we can identify entry points for policies and interventions, and in some countries awareness of the problem among policy makers and public health practitioners has even produced an urge to implement policies and interventions that can reasonably be expected to be effective. Examples of such countries include the Netherlands, Sweden, and the United Kingdom, but more are likely to follow, given the current worldwide interest in inequalities in health.

Unfortunately, it is exceedingly difficult to find good evidence on the effectiveness of policies and interventions to reduce inequalities in health. The experience of both the Acheson Committee, conducting the Independent Inquiry into Inequalities in Health in Britain, and the Albeda Committee, developing a programme to reduce inequalities in health for the Dutch government, illustrate this. This lack of evidence is the result of a combination of factors: historically, very few policies and interventions have been implemented that explicitly targeted inequalities in health; many policies and interventions that were implemented have not been evaluated; those that were evaluated were not evaluated adequately; and results of relevant evaluation studies are often not easily accessible to policy makers and public health practitioners.

There is no reason why this situation could not be changed for the better. The "experience base" in which evaluation studies could be conducted is not small but huge, if we agree that much can be learned from policies and interventions that try to improve the living conditions and life chances of people with socioeconomic disadvantage. These can be found everywhere, and the trick may just be to add health measurements to ongoing evaluation efforts. Many clinical and public health trials are conducted in socioeconomically heterogeneous populations, and analyses by socioeconomic subgroup may tell us more about what works and what not in these groups. And, finally, there is a lot of work going on in many countries to improve the health of socioeconomically disadvantaged groups that is simply not evaluated at all. Even modest evaluation efforts, focusing on process instead of outcome measures and using quasi-experimental instead of fully experimental designs, if properly conducted, can generate useful knowledge, and are certainly better than doing nothing to evaluate the effectiveness of policies and interventions.

Accessibility of results of evaluation studies can be improved by creating clearing houses and review groups. A recent overview of the European experience with policies and interventions to reduce inequalities in health ${ }^{1}$ has shown again that accessibility is an enormous problem. It is difficult to find relevant studies in conventional literature databases, because keyword systems do not adequately identify such studies, and because some of these studies are not included in these databases, for example, because they are only reported in the national literature or "grey reports". International exchanges such as the one just mentioned typically identify a wide range of experiences, and uncover evaluation studies that have not reached the international literature. We therefore need a special effort to increase the accessibility of evaluation results, for example in the form of clearing houses. These may, on the basis of carefully designed search strategies and an international reporting system to collect information on ongoing and recently completed evaluation studies, increase the accessibility to policy makers and public health practitioners of relevant information. Another problem relates to the methodological evaluation of the evidence presented in the literature. Because of the difficulties in applying traditional research methods in this area many studies use alternative designs that are sometimes difficult to evaluate. It would therefore be useful to have international review and methods groups for policies and interventions to reduce inequalities in health, by analogy to or as part of the Cochrane or Campbell Collaborations, that could help in building a systematic evidence base.

\section{J P Mackenbach,}

Department of Public Health, Erasmus MC, University Medical Centre, Rotterdam, Netherlands; mackenbach@mgz.fgg.eur.nl

1 Mackenbach JP, Bakker $\mathrm{W}$, eds. Reducing inequalities in health: the European experience. London: Routledge, 2002. 